\title{
High levels of chemerin associated with variants in the NOS3 and $A P O B$ genes in rural populations of Ouro Preto, Minas Gerais, Brazil
}

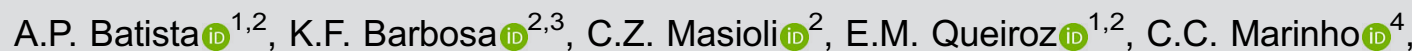 \\ A.P.C. Cândido ${ }^{5}{ }^{5}$, and G.L.L. Machado-Coelho ${ }^{10}{ }^{1,2}$ \\ ${ }^{1}$ Núcleo de Pesquisa em Ciências Biológicas, Programa de Pós-Graduação em Ciências Biológicas, Instituto de Ciências Exatas e \\ Biológicas, Universidade Federal de Ouro Preto, Ouro Preto, MG, Brasil \\ ${ }^{2}$ Laboratório de Epidemiologia, Departamento de Medicina de Família, Saúde Mental e Coletiva, Escola de Medicina, Universidade \\ Federal de Ouro Preto, Ouro Preto, MG, Brasil \\ ${ }^{3}$ Programa de Pós-Graduação em Saúde e Nutrição, Escola de Nutrição, Universidade Federal de Ouro Preto, \\ Ouro Preto, MG, Brasil \\ ${ }^{4}$ Departamento de Clínica Médica, Faculdade de Medicina, Universidade Federal de Minas Gerais, Belo Horizonte, MG, Brasil \\ ${ }^{5}$ Departamento de Nutrição, Instituto de Ciências Biológicas, Universidade Federal de Juiz de Fora, Juiz de Fora, MG, Brasil
}

\begin{abstract}
Chemerin is an adipokine that has been associated with components of metabolic syndrome. It has been described to affect adipocyte metabolism and inflammatory responses in adipose tissue, as well as the systemic metabolism of lipids and glucose. Few epidemiological studies have evaluated classical and genetics cardiovascular risk factors (CVRFs) in the mixed adult rural population in Brazil. Therefore, the present study explored possible associations between CVRFs and chemerin. This crosssectional study included 508 adults from the rural localities of Lavras Novas, Chapada, and Santo Antônio do Salto in Ouro Preto, Minas Gerais, Southeast Brazil. Demographic, behavioral, clinical, biochemical, anthropometric variables, and 12 single nucleotide polymorphisms (SNPs) linked with metabolic syndrome phenotypes were evaluated for associations with chemerin level. There was a significant association of high triglyceride levels [odds ratio $(\mathrm{OR})=1.91,95 \% \mathrm{Cl}: 1.23-2.98$ ], insulin resistance $(\mathrm{OR}=1.82$, 95\% Cl: $1.03-3.22)$, age $(\mathrm{OR}=1.64,95 \% \mathrm{Cl}: 1.08-2.49)$, and sex (OR=1.99, 95\%Cl: 1.35-2.95) with high levels of chemerin. High chemerin levels were significantly associated with the genetic polymorphisms rs693 in the APOB gene $(\mathrm{OR}=1.50,95 \% \mathrm{Cl}: 1.03-2.19)$ and $\mathrm{rs} 1799983$ in the $N O S 3$ gene $(\mathrm{OR}=1.46,95 \% \mathrm{Cl}: 1.01-2.12)$ for the AA and GT + TT genotypes, respectively. In the concomitant presence of genotypes AA of rs693 and GT+ TT of rs1799983, the chance of presenting high levels of chemerin showed a 2.21 -fold increase $(95 \% \mathrm{Cl}: 1.25-3.88)$ compared to the reference genotype. The development of classical CVRFs in this population may be influenced by chemerin and by two risk genotypes characteristic of variants in well-studied genes for hypertension and dyslipidemia.
\end{abstract}

Key words: Genetic epidemiology; Adipokines; Dyslipidemia; Obesity; Diabetes; Hypertension

\section{Introduction}

Over the last few decades, cardiovascular disease (CVD) has been recognized as one of the world's leading causes of death (1). The burden of CVD can be projected in terms of risk factors, often portrayed as modifiable or non-modifiable (1).

In addition to the classical risk factors, new biomarkers of cardiovascular risk have been explored. Among them is chemerin, which is an adipokine also known as RARRES2 (retinoic acid receptor-responding protein 2). It was originally described as a chemoattractant for cells of the immune system (2). Since 2007, both chemerin and its receptor CMKLR1 have been found to be strongly expressed in white, visceral, and subcutaneous adipose tissue from animal models and in humans. Subsequently, chemerin was identified as a new adipokine with potential autocrine and paracrine functions $(2,3)$. In recent years, several studies have discussed its role as a cardiovascular risk biomarker considering its association with components of metabolic syndrome, including obesity and overweight (MIM: 601665), type 2 diabetes mellitus (T2DM) (MIM: 125853), dyslipidemias (MIM: 143890), and arterial hypertension (AH) (MIM: 145500) $(2,4,5)$. 
In addition to clinical and biochemical cardiovascular risk factors (CVRFs), research has steadily improved the understanding of the genetic basis of complex human diseases. Remarkable advances have been made in the discovery of new genetic associations with classical CVRFs such as dyslipidemias, AH, T2DM, and obesity (6).

Ouro Preto, Minas Gerais (MG) is located in the southeast region of Brazil. Its population is formed by the miscegenation of Europeans, Africans, and Amerindians, historically gathered by the economic activity based on gold extraction. In 2001, the study "Hearts of Ouro Preto" described a disturbingly high prevalence of classical CVRFs such as $\mathrm{AH}$, hyperglycemia, dyslipidemia, obesity, and central obesity in the adult urban population of Ouro Preto $(7,8)$, as well as in children and adolescents (9), emphasizing that prompt action was required.

To date, few epidemiological studies have evaluated classical and emerging risk factors in the mixed adult rural population in Brazil. Therefore, the present study explored possible associations between classical CVRFs and chemerin. The objective was to evaluate it as a candidate predictor of cardiovascular risk in two rural localities of Ouro Preto. In addition, a panel of 12 single nucleotide polymorphisms (SNPs) was genotyped in genes previously described as modulators of cardiovascular risk, including the chemerin-encoding RARRES2 gene, to evaluate whether chemerin is associated with genetic variants.

\section{Material and Methods}

\section{Ethical aspects}

This study was approved by the Human Research Ethics Committee of the Federal University of Ouro Preto (No. 125017/2015 and CAAE 51666115.5.0000.5150). All participants provided written consent after receiving broad information.

\section{Study design and population}

A population-based cross-sectional study was carried out in two rural districts of Ouro Preto, namely Lavras Novas, including its subdistrict Chapada, and Santo Antônio do Salto, between May 2015 and December 2016. Men and women $>18$ years and living in the localities for at least 5 years were considered eligible. Adults who did not complete the questionnaire, did not attend the collection of blood samples, or refused to provide written informed consent were not included.

\section{Sample size and sampling process}

A sample size of 600 subjects, including $10 \%$ losses, was defined based on the following assumptions: study population of 1509 subjects, confidence level of 95\%, study power of $99 \%$, expected frequency of $\mathrm{AH}$ in the population $38 \%$, and expected error of $5 \%$. The sampling process was based on stratification by sex and age group, where all eligible residents were invited to participate in the study. A total of 508 participants were included in the analysis.

From a total of 1509 individuals eligible for the present study, 847 people refused consent or were absent from the home where the questionnaire was applied. Of the 662 who responded to the questionnaire, 154 did not attend the blood collection session, totaling 508 participants (Figure 1). The losses were non-differential by sex and greater in the elderly.

\section{Homogeneity of the sample in relation to losses}

In order to observe the homogeneity of the sample regarding the representativeness of the population among the refusals, permission was requested to gauge the blood pressure, and after analysis, there was no significant difference between the participants and the refusal group $(P=0.558)$. The mean systolic blood pressure (SBP) and diastolic blood pressure (DBP) in the participating group were $130.8 \mathrm{mmHg}( \pm 22.7)$ and $79.6 \mathrm{mmHg}$ ( \pm 14.6$)$, respectively, whereas in the refusal group, they were $133.1 \mathrm{mmHg}( \pm 16.0)$ and $83.8 \mathrm{mmHg}( \pm 12.6)$, respectively.

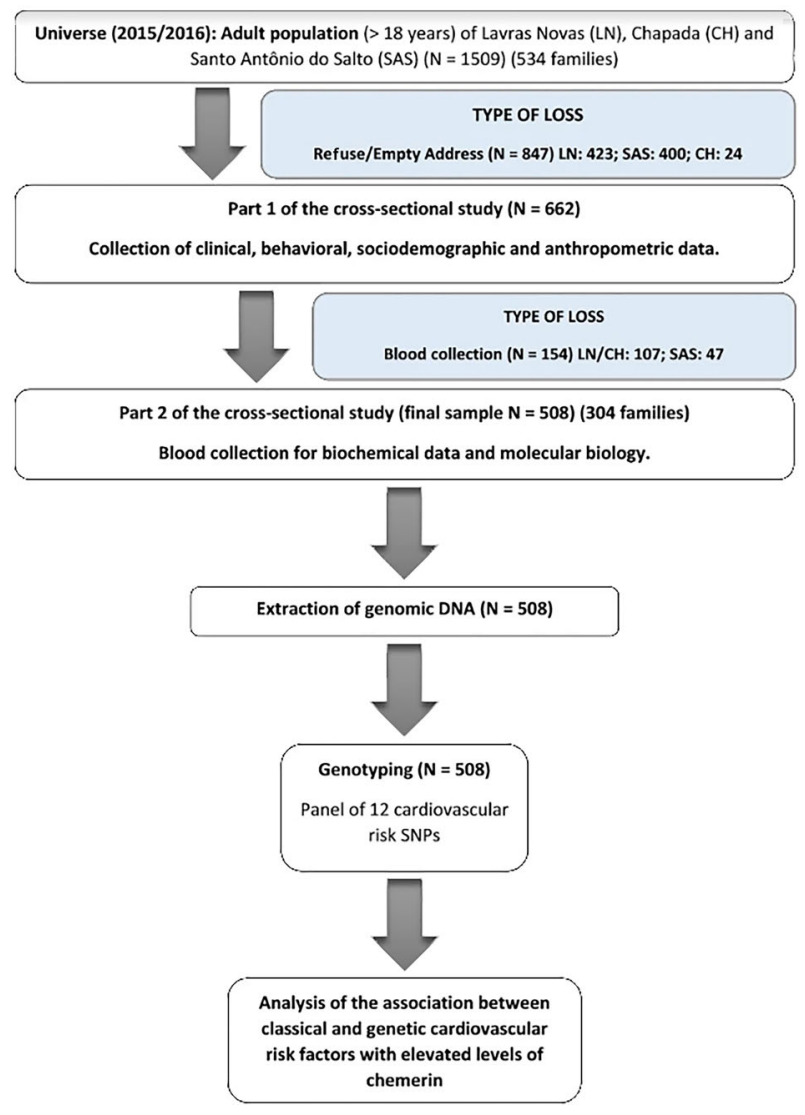

Figure 1. Sampling process and analysis stages of the present study. SNP: single nucleotide polymorphisms. 


\section{Exposure, outcome, and confounders}

Demographic, behavioral, clinical, biochemical, and anthropometric variables and 12 single nucleotide polymorphisms (SNPs) linked with metabolic syndrome phenotypes were evaluated as exposure. The levels of chemerin above the median were considered the outcome. Sex, age, and biochemical measurements were considered the main confounders.

\section{Sociodemographic and behavioral data}

The sociodemographic characteristics (sex, age, income, marital status, education, and self-reported skin color), behavioral characteristics (smoking and alcohol consumption), and medication use were recorded in a semi-structured questionnaire filled in by trained interviewers. The classification of smoking status was based on self-reported past and/or current use of cigarettes. Three groups were considered: 1) never smokers: those who did not smoke or smoked less than 100 cigarettes throughout their lives; 2) ex-smokers: those who smoked at least 100 cigarettes during their lifetime but stopped smoking for more than 6 months; and 3) current smokers: those who had smoked 100 or more cigarettes during their lifetime and continued to smoke (10). For statistical analysis, current smokers and ex-smokers were grouped as 'smokers'. The degree of dependence on alcohol drinking was evaluated using the Cut-down, Annoyed, Guilty, Eyeopener (CAGE) questionnaire (11).

\section{Risk factors for cardiovascular disease}

The following classical CVRFs were evaluated independently: $\mathrm{AH}, \mathrm{T} 2 \mathrm{DM}$, obesity, insulin resistance (IR), and dyslipidemia (data not shown).

Blood pressure. Three measures of blood pressure were taken at 1-2-min intervals with a $\mathrm{HEM}-705 \mathrm{CP}^{\circledR}$ automatic digital blood pressure monitor (Omron Healthcare, USA). The participant was kept in a seated position, and the cuff was firmly fit and maintained at heart level. The lowest blood pressure measure was considered (12). Subjects who were on antihypertensives and/or had a casual measurement $\geqslant 140 / 90 \mathrm{mmHg}$ were considered hypertensives (12). When SBP and DBP were in different categories, the highest category was used for classification.

Height and body weight. Stature was measured in centimeters $(\mathrm{cm})$ with a portable stadiometer (Charder, Taiwan). The participant was requested to dress in light clothing and to stand barefoot with heels together in the center of the scale with his/her back to the marker, arms extended along the body, eyes fixed forward, erect head, and touching the vertical bar of the stadiometer (13). Body weight was measured with a TANITA ${ }^{\mathbb{R}}$ (USA) portable scale with a maximum capacity of $150 \mathrm{~kg}$ and precision of $0.1 \mathrm{~kg}(13)$.

Body mass index (BMI). BMI was calculated as weight $(\mathrm{kg}) /$ stature $(\mathrm{m})^{2}$ and classified as 'normal' $\left(<25 \mathrm{~kg} / \mathrm{m}^{2}\right)$, 'overweight' $\left(25-29.9 \mathrm{~kg} / \mathrm{m}^{2}\right)$ and 'obese' $\left(\geqslant 30 \mathrm{~kg} / \mathrm{m}^{2}\right)$ (14).

Waist circumference (WC). WC was measured with a simple inelastic tape. The participants were requested to stand upright, abdomen relaxed, arms extended laterally along the body, feet slightly apart, and weight equally distributed between the legs. Cut-off points established for Brazilian adults were used for the classification of WC, taking cardiovascular risk as an outcome (15). Cut-off points at $87 \mathrm{~cm}$ for women and $95 \mathrm{~cm}$ for men were considered for cardiovascular risk.

\section{Blood biochemistry and molecular analysis}

Lipid profile. The enzymatic-colorimetric method was used for the concentration of triglycerides (TG), total cholesterol, and high-density lipoprotein (HDL-c) fractions (Triglycerides Liquicolor Mono ${ }^{\circledR}$, Cholesterol Liquicolor $^{\circledR}$, HDL Cholesterol Direct/Homogeneous Direct Test ${ }^{\mathbb{R}}$, all Invitro Diagnostic/Human, Brazil; Automatic Analyzer Chemwell R6 ${ }^{\circledR}$, Awareness Technology, USA). Low-density lipoprotein (LDL-C) and very low-density lipoprotein (VLDL-c) fractions were calculated with Friedwald's formula (1972) (16): LDL-c (mg/dL) = total cholesterol - HDL-c - (triglycerides/5) and VLDL-c(mg/ $\mathrm{dL})=$ triglycerides $/ 5$, when the triglyceride concentration was $\leqslant 400 \mathrm{mg} / \mathrm{dL}$.

Definition of dyslipidemia. Dyslipidemias were classified according to the increased lipid fraction as follows: isolated hypercholesterolemia: isolated increase in LDL-C $\geqslant 160 \mathrm{mg} / \mathrm{dL}$; isolated hypertriglyceridemia: isolated TG increase $\geqslant 150 \mathrm{mg} / \mathrm{dL}$; mixed hyperlipidemia: increased LDL-c $\geqslant 160 \mathrm{mg} / \mathrm{dL} ; \mathrm{TG} \geqslant 150 \mathrm{mg} / \mathrm{dL}$; and HDL-c: reduction of $\mathrm{HDL}-\mathrm{C}<40 \mathrm{mg} / \mathrm{dL}$ for men and $<50 \mathrm{mg} / \mathrm{dL}$ for women, alone or in combination with increased LDL-c or TG (17). Dyslipidemia was defined as an increase in TG or LDL-c or total cholesterol $\geqslant 190 \mathrm{mg} / \mathrm{dL}$, reduction in HDL-c, or any combination of the above.

Fasting glycemia. Fasting glycemia was measured by enzymatic colorimetric analysis without deproteinization (Enzyme Glucose, Invitro Diagnostic/Human, Brazil; Chemwell R6 ${ }^{\circledR}$ Automatic Analyzer, Awareness Technology, USA). Glycemia was classified as 'normal' (<100 $\mathrm{mg} / \mathrm{dL})$, 'altered fasting glycemia' (100-125.9 mg/dL), and 'hyperglycemia' ( $\geqslant 126 \mathrm{mg} / \mathrm{dL}$ ) (18).

Definition of diabetes. Individuals on oral hypoglycemic medication or insulin and/or fasting glycemia $\geqslant 126$ $\mathrm{mg} / \mathrm{dL}$ were considered diabetic (18). Prediabetic subjects (100-125.9 mg/dL) who did not use hypoglycemic drugs were not considered diabetic.

Fasting insulinemia (Fl). FI was measured by indirect chemiluminescence according to the manufacturer's protocol (Access 2 Immunoassay System ${ }^{\mathbb{B}}$, Beckman Coulter, USA).

Insulin resistance. IR was determined by the Homeostatic Model Assessment of IR (HOMA-IR) index with the 
formula FI $(\mu \mathrm{Ul} / \mathrm{mL}) \times$ fasting glycemia $(\mathrm{mM}) / 22.5(19)$. The cut-off for IR in adults was 2.71 (20).

Chemerin. Blood levels of chemerin were measured with an enzyme-linked immunosorbent assay (ELISA) for human capture (Abcam Kit, Chemwell R6 ${ }^{\circledR}$ Automatic Analyzer, Awareness Technology, USA). The results were divided into medians for pooled analysis.

Extraction of genomic DNA. Genomic DNA extraction was performed with the Wizard ${ }^{\circledR}$ Genomic DNA Purification Kit (Promega ${ }^{\circledR}$, USA) according to the manufacturer's protocol.

\section{Analysis of genetic markers of cardiovascular risk}

The allelic and genotypic frequencies of a panel of 12 single nucleotide polymorphisms (SNPs) were evaluated and tested for Hardy-Weinberg equilibrium. The criteria for choosing the panel involved the following: 1) present a positive association with CVRFs (AH, obesity, dyslipidemias, T2DM, and IR) in at least five previous studies, with at least one being a Genome Wide Association Study (GWAS), and in ethnic groups similar to the study population; and 2) not present rare alleles in studies with African and European populations. The definition and characteristics of the SNPs are reported in Table 1 (5,21-27).

Regarding the SNPs rs429358 and rs7412 in the $A P O E$ gene, there is the peculiarity that the combination of these characterizes a haplotype that generates three common variants called $\epsilon 2, \epsilon 3$, and $\epsilon 4$ (28). Also the combination of the alleles APOE rs429358 and rs7412 identify the isoforms: $€ 2$ or $\mathrm{APOE}{ }^{*} 2, \epsilon 3$ or $\mathrm{APOE}{ }^{*} 3, \epsilon 4$ or APOE *4.

The allelic discrimination technique was performed by real-time PCR (qPCR) using a set of primers and probes specific for each SNP (TaqMan ${ }^{\circledR}$ Minor Groove BinderMGB, TaqMan ${ }^{\circledR}$ System; 7500 Fast Real-Time PCR Systems, Applied Biosystems, USA).

\section{Statistical analysis}

The database was constructed and analyzed using SPSS software version 22 (IBM, USA). The prevalence was calculated by the ratio between the number of cases for each CVRF and the total number of individuals in the sample. The allele frequencies were obtained by gene counting and tested for Hardy-Weinberg equilibrium (with the exception of the SNPs rs429358 and rs7412 APOE gene). Continuous variables were tested for normality of distribution by the Shapiro-Wilk test. For those with a normal distribution, one-way analysis of variance (ANOVA) was performed. Categorical variables were analyzed using Pearson's chi-squared or Fisher's exact test. The distribution of panel genotypes of 12 single nucleotide polymorphisms (SNPs) between the groups of chemerin quartiles was tested with Pearson's chi-squared test. The binary logistic regression analysis was used to adjust associations for confounders $(P<0.20)$, and the odds ratio $(O R)$ was calculated to identify the genetic and classical markers associated with high levels of chemerin in a significant $(P<0.05)$ and independent way.

\section{Results}

A total of 329 women $(64.8 \%$, mean age $49.2 \pm 17.3)$ and 179 men $(35.2 \%$, mean age $49.9 \pm 16.6)$ participated. The age range was 18-91 years. Twenty participants (3.9\%) lived in Chapada, 189 (36.7\%) lived in Lavras Novas, and 306 (59.4\%) lived in Santo Antônio do Salto. Table 2 shows the sociodemographic and behavioral characteristics of the study population and the levels of chemerin grouped in quartiles. Elderly, female, and illiterate groups were more likely to present higher levels of chemerin. Regarding marital status, higher levels of chemerin were found in married or in stable unions, followed by widowed and divorced participants.

Table 1. Panel characteristics of 12 single nucleotide polymorphisms (SNPs) selected for the study.

\begin{tabular}{|c|c|c|c|c|c|}
\hline Phenotype & RefSeqGene HGVS/refSNP & Chromosome & Gene/HGNC ID & Allele described in dbSNP & Reference \\
\hline \multirow[t]{3}{*}{ Hypertension } & g.9543T > C/rs699 & $1 q 42.2$ & $A G T / 333$ & $\mathrm{C} / \mathrm{T}$ (REV) & 21 \\
\hline & g.12965T> G/rs1799983 & $7 q 36.1$ & NOS3/7876 & $\mathrm{G} / \mathrm{T}(\mathrm{FWD})$ & 21 \\
\hline & g. $10501 \mathrm{C}>\mathrm{T} / \mathrm{rs} 5443$ & $12 \mathrm{p} 13.31$ & GNB3/4400 & $\mathrm{C} / \mathrm{T}$ (FWD) & 21 \\
\hline \multirow[t]{6}{*}{ Dyslipidemia } & g.7903T > C/rs429358 & $19 q 13.32$ & APOE/613 & $\mathrm{C} / \mathrm{T}(\mathrm{FWD})$ & 22 \\
\hline & g.8041C > T/rs7412 & $19 q 13.32$ & APOE/613 & $\mathrm{C} / \mathrm{T}(\mathrm{FWD})$ & 22 \\
\hline & g. $39751 \mathrm{C}>\mathrm{T} / \mathrm{rs} 693$ & $2 \mathrm{p} 24.1$ & $A P O B / 603$ & $\mathrm{G} / \mathrm{A}(\mathrm{FWD})$ & 23 \\
\hline & g.5912T > C/rs4520 & $11 q 23.3$ & APOC $3 / 610$ & $\mathrm{C} / \mathrm{T}(\mathrm{FWD})$ & 24 \\
\hline & g. $8017 \mathrm{G}>\mathrm{C} / \mathrm{rs} 5128$ & $11 q 23.3$ & APOC $3 / 610$ & $\mathrm{C} / \mathrm{G}(\mathrm{REV})$ & 25 \\
\hline & g. $35825 \mathrm{~T}>\mathrm{C} / \mathrm{rs} 5925$ & $19 p 13.2$ & $L D L R / 6547$ & $\mathrm{C} / \mathrm{T}(\mathrm{FWD})$ & 26 \\
\hline \multirow[t]{3}{*}{ Obesity, IR, and T2DM } & g.68777C > G/rs1801282 & $3 p 25.2$ & PPARG/9236 & C/G (FWD) & 27 \\
\hline & c. $515+281 \mathrm{~T}>\mathrm{G} / \mathrm{rs} 4721$ & $7 q 36.1$ & RARRES2/9868 & $\mathrm{A} / \mathrm{C}(\mathrm{REV})$ & 5 \\
\hline & c. $280-494 \mathrm{~T}>\mathrm{G} / \mathrm{rs} 17173608$ & $7 q 36.1$ & RARRES2/9868 & $\mathrm{G} / \mathrm{T}(\mathrm{FWD})$ & 5 \\
\hline
\end{tabular}

IR: insulin resistance; T2DM: type 2 diabetes mellitus; HGVS: Human Genome Variation Society; HGNC: HUGO Gene Nomenclature Committee. 
Table 2. Socio-demographic and behavioral characteristics of the study population by quartiles of chemerin.

\begin{tabular}{|c|c|c|c|c|c|}
\hline \multirow[t]{2}{*}{ Variables } & \multicolumn{3}{|c|}{ Chemerin (ng/mL) } & \multirow[t]{2}{*}{ OR $(95 \% \mathrm{Cl})$} & \multirow[t]{2}{*}{$\mathrm{P}^{*}$} \\
\hline & $<160(\mathrm{n}=255)$ & $\geqslant 160(n=253)$ & Total $(n=508)$ & & \\
\hline \multicolumn{6}{|l|}{ Age group } \\
\hline 18-34 years & $84(32.9)$ & $67(26.5)$ & $151(29.7)$ & 1.0 & \\
\hline $35-59$ years & $113(44.3)$ & $104(41.1)$ & $217(42.7)$ & $1.15(0.76-1.75)$ & 0.501 \\
\hline$\geqslant 60$ years & $58(22.8)$ & $82(32.4)$ & $140(27.6)$ & $1.77(1.11-2.82)$ & 0.015 \\
\hline \multicolumn{6}{|l|}{ Gender } \\
\hline Male & $111(43.5)$ & $68(26.9)$ & $179(35.2)$ & 1.0 & \\
\hline Female & $144(56.5)$ & $185(73.1)$ & $329(64.8)$ & $2.09(1.44-3.04)$ & $<0.0001$ \\
\hline \multicolumn{6}{|l|}{ Schooling } \\
\hline Literate & $241(94.5)$ & $224(88,9)$ & $465(91.7)$ & 1.0 & \\
\hline Illiterate & $14(5.5)$ & $28(11.1)$ & $42(8.3)$ & $2.15(1.10-4.19)$ & 0.022 \\
\hline Missing data** & 0 & 1 & 1 & & \\
\hline \multicolumn{6}{|l|}{ Marital status } \\
\hline Not married & $84(32,9)$ & $51(20.2)$ & $135(26.6)$ & 1.0 & \\
\hline Married or SU & $146(57.3)$ & $163(64.7)$ & $309(60.9)$ & $1.85(1.22-2.8)$ & 0.003 \\
\hline Others $^{a}$ & $25(9.8)$ & $38(15.1)$ & $63(12.4)$ & $2.5(1.35-4.62)$ & 0.002 \\
\hline Missing data** & 0 & 1 & 1 & & \\
\hline \multicolumn{6}{|l|}{ Income } \\
\hline$\geqslant 4 \mathrm{MS}$ & $27(11.1)$ & $40(16.6)$ & $67(13.8)$ & 1.0 & \\
\hline 2 a $3 \mathrm{MS}$ & $147(60.5)$ & $122(50.6)$ & $269(55.6)$ & $0.56(0.32-0.96)$ & 0.035 \\
\hline$\leqslant 1 \mathrm{MS}$ & $69(28.4)$ & 79 (32.8) & $148(30.6)$ & $0.77(0.42-1.38)$ & 0.387 \\
\hline Missing data** & 12 & 12 & 24 & & \\
\hline \multicolumn{6}{|l|}{ Self-reported skin color } \\
\hline White + yellow & $31(12.4)$ & $31(12.3)$ & $62(12.3)$ & 1.0 & \\
\hline Black + brown & $220(87.6)$ & $221(87.7)$ & $441(87.7)$ & $1.0(0.59-1.71)$ & 0.987 \\
\hline Missing data** & 4 & 1 & 5 & & \\
\hline \multicolumn{6}{|l|}{ Smoking $^{\mathrm{b}}$} \\
\hline No & $158(62.7)$ & $162(64.8)$ & $320(63.7)$ & 1.0 & \\
\hline Yes & $94(37.3)$ & $88(35.2)$ & $182(36.3)$ & $0.91(0.63-1.31)$ & 0.642 \\
\hline Missing data** & 3 & 3 & 6 & & \\
\hline \multicolumn{6}{|l|}{ Alcohol drinking $^{c}$} \\
\hline Low risk/no alcohol drinking & $227(89.7)$ & $226(93.4)$ & $453(91.5)$ & 1.0 & \\
\hline Alcohol dependence & $26(10.3)$ & $16(6.6)$ & $42(8.5)$ & $0.61(0.32-1.18)$ & 0.144 \\
\hline Missing data** & 2 & 11 & 13 & & \\
\hline
\end{tabular}

Data are reported as number and percent. SU: stable union; MS: minimum salary. ${ }^{*} \mathrm{P}<0.05$ (Pearson's chi-square test). ${ }^{* *}$ Missing data were excluded from analysis; ${ }^{a}$ separated/divorced or widowed; ' self-reported lifetime and/or current smoking; ${ }^{\mathrm{c}}$ according to the CAGE (Cut-down, Annoyed, Guilty, Eye-opener) questionnaire.

The anthropometric, biochemical, and clinical characteristics according to quartiles of chemerin levels are shown in Table 3. There was a significant difference in chemerin levels between individuals with central obesity, high TG, and dyslipidemia than the reference group. In addition, altered glycemia, insulin levels above p75, and IR were associated with higher levels of chemerin.

Table 4 presents the analysis of the allelic and genotypic frequencies of a panel of 12 genetic markers of the SNP type by quartiles of chemerin level. Levels were significantly higher in the presence of the GT genotype rs1799983 (g.12965T>G) of the NOS3 gene and the AA genotype rs693 (g.39751C > T) polymorphism of the $A P O B$ gene compared to the respective ancestral homozygotes (GG for both).

The multivariate analysis was performed by binary logistic regression adjusting the levels of chemerin by the anthropometric, body composition, biochemical, sociodemographic, behavioral, and genetic variables. Table 5 shows the variables that remained significant after adjustment.

Table 6 shows the analysis of risk adjusted for sex, age, HOMA-IR, TG, and HDL-c for the chemerin groups in relation to the marker genotypes rs693 and rs 1799983 . 
Table 3. Anthropometric, clinical, and biochemical characteristics of the study population by quartiles of chemerin.

\begin{tabular}{|c|c|c|c|c|c|}
\hline \multirow[t]{2}{*}{ Variables } & \multicolumn{3}{|c|}{ Chemerin (ng/mL) } & \multirow[t]{2}{*}{ OR $(95 \% \mathrm{Cl})$} & \multirow[t]{2}{*}{$\mathrm{P}^{*}$} \\
\hline & $<160(n=255)$ & $\geqslant 160(n=253)$ & Total $(n=508)$ & & \\
\hline \multicolumn{6}{|l|}{ BMI $\left(\mathrm{kg} / \mathrm{m}^{2}\right)$} \\
\hline$<25$ & $123(48.6)$ & $101(40.1)$ & $224(44.4)$ & 1.0 & 0.054 \\
\hline$\geqslant 25$ & $130(51.4)$ & $151(59.9)$ & $281(55.6)$ & $1.45(0.99-2.01)$ & \\
\hline Missing data** & 2 & 1 & 3 & & \\
\hline \multicolumn{6}{|l|}{ WC $(\mathrm{cm})$} \\
\hline Normal & $157(62.3)$ & $120(48.2)$ & $277(55.3)$ & 1.0 & 0.001 \\
\hline Cardiovascular risk & $95(37.7)$ & $129(51.8)$ & $224(44.7)$ & $1.77(1.24-2.53)$ & \\
\hline Missing data** & 2 & 5 & 7 & & \\
\hline \multicolumn{6}{|l|}{ TG (mg/dL) } \\
\hline$<150$ & $208(81.6)$ & $171(67.6)$ & $379(74.6)$ & 1.0 & $<0.0001$ \\
\hline$\geqslant 150$ & $47(18.4)$ & $82(32.4)$ & $129(25.4)$ & $2.12(1.40-3.2)$ & \\
\hline \multicolumn{6}{|l|}{ TC (mg/dL) } \\
\hline$<190$ & $141(55.3)$ & $120(47.4)$ & $261(51.4)$ & 1.0 & 0.076 \\
\hline$\geqslant 190$ & $114(44.7)$ & $133(52.6)$ & 247 (48.6) & $1.37(0.96-1.94)$ & \\
\hline \multicolumn{6}{|l|}{ LDL-c (mg/dL) } \\
\hline$<130$ & $207(81.2)$ & $201(79.4)$ & $408(80.3)$ & 1.0 & 0.624 \\
\hline$\geqslant 130$ & $48(18.8)$ & $52(20.6)$ & $100(19.7)$ & $1.11(0.72-1.72)$ & \\
\hline \multicolumn{6}{|l|}{ HDL-c (mg/dL) } \\
\hline$\geqslant 40$ & $231(90.6)$ & $225(88.9)$ & $456(89.8)$ & 1.0 & 0.0538 \\
\hline$<40$ & $24(9.4)$ & $28(11.1)$ & $52(10.2)$ & $1.19(0.67-2.12)$ & \\
\hline \multicolumn{6}{|l|}{ Dyslipidemia } \\
\hline No & $184(72.2)$ & $151(59.7)$ & 335 (65.9) & 1.0 & 0.003 \\
\hline Yes & $71(27.8)$ & $102(40.3)$ & $173(34.1)$ & $1.75(1.20-2.53)$ & \\
\hline \multicolumn{6}{|l|}{$\mathrm{FG}(\mathrm{mg} / \mathrm{dL})$} \\
\hline$<100$ & $136(53.3)$ & $102(40.3)$ & $238(46.9)$ & 1.0 & \\
\hline $100-125$ & $107(42.0)$ & $123(48.6)$ & $230(45.2)$ & $1.53(1.06-2.20)$ & 0.021 \\
\hline$>125$ & $12(4.7)$ & $28(11.1)$ & $40(7.9)$ & $3.11(1.50-6.41)$ & 0.001 \\
\hline \multicolumn{6}{|l|}{$\mathrm{FI}(\mu \mathrm{LU} / \mathrm{mL})$} \\
\hline$<\mathrm{p} 75$ & $207(81.2)$ & $174(68.8)$ & $381(75.0)$ & 1.0 & 0.001 \\
\hline$\geqslant p 75$ & $48(18.8)$ & $79(31.2)$ & $127(25.0)$ & $1.95(1.29-2.95)$ & \\
\hline \multicolumn{6}{|l|}{ HOMA-IR } \\
\hline$<2.71$ & $231(90.6)$ & $203(80.2)$ & $434(85.4)$ & 1.0 & 0.001 \\
\hline$\geqslant 2.71$ & $24(9,4)$ & $50(19.8)$ & $74(14.6)$ & $2.37(1.40-3.95)$ & \\
\hline \multicolumn{6}{|l|}{ Diabetes } \\
\hline No & $239(93.7)$ & $220(87.0)$ & $459(90.4)$ & 1.0 & 0.01 \\
\hline Yes & $16(6.3)$ & $33(13.0)$ & $49(9.6)$ & $2.24(1.20-4.18)$ & \\
\hline \multicolumn{6}{|l|}{ Hypertension } \\
\hline No & $151(59.2)$ & $140(55.3)$ & $291(57.3)$ & 1.0 & 0.377 \\
\hline Yes & $104(40.8)$ & $113(44.7)$ & $217(42.7)$ & $1.17(0.82-1.66)$ & \\
\hline \multicolumn{6}{|l|}{$\mathrm{SBP}(\mathrm{mmHg})$} \\
\hline Normal/borderline & $165(64.7)$ & $164(64.8)$ & $329(64.8)$ & 1.0 & 0.978 \\
\hline High & $90(35.3)$ & $89(35.2)$ & $179(35.2)$ & $0.99(0.69-1.43)$ & \\
\hline \multicolumn{6}{|l|}{$\mathrm{DBP}(\mathrm{mmHg})$} \\
\hline Normal/borderline & $206(80.8)$ & $204(80.6)$ & $410(80.7)$ & 1.0 & 0.965 \\
\hline High & 49 (19.2) & $49(19.4)$ & $98(19.3)$ & $1.01(0.65-1.56)$ & \\
\hline
\end{tabular}

Data are reported as number and percent. ${ }^{*} \mathrm{P}<0.05$ (Pearson's chi-squared test). ${ }^{*}$ Missing data were excluded from analysis; BMI: body mass index; WC: waist circumference; TG: triglycerides; TC: total cholesterol; LDL-C: low-density lipoprotein; HDL-C: high-density lipoprotein; FG: fasting glycemia; FI: fasting insulinemia; HOMA-IR: Homeostatic Model Assessment of Insulin Resistance; SBP: systolic blood pressure; DBP: diastolic blood pressure. 
Table 4. Frequency of genotypes of a panel of 12 single nucleotide polymorphisms (SNP)-like polymorphisms in the study population by quartiles of chemerin.

\begin{tabular}{|c|c|c|c|c|c|c|}
\hline \multirow[t]{2}{*}{ Gene/dbSNP rs ID } & \multirow[t]{2}{*}{ Genotypes* } & \multicolumn{3}{|c|}{ Chemerin (ng/mL) } & \multirow[t]{2}{*}{ OR $(95 \% \mathrm{Cl})$} & \multirow[t]{2}{*}{$P^{* *}$} \\
\hline & & $<160(n=255)$ & $\geqslant 160(n=253)$ & Total $(n=508)$ & & \\
\hline \multirow[t]{3}{*}{ PPARG/rs1801282 } & $\mathrm{CC}$ & $248(97.3)$ & $247(97.6)$ & $495(97.4)$ & 1.0 & \\
\hline & CG & $7(2.7)$ & $6(2.4)$ & $13(2.6)$ & $0.86(0.28-2.59)$ & 0.790 \\
\hline & GG & - & - & - & & \\
\hline \multirow[t]{3}{*}{ RARRES2/rs4721 } & GG & $61(23.9)$ & 49 (19. 3) & $110(21.7)$ & 1.0 & \\
\hline & GT & $116(45.5)$ & $134(53.0)$ & $250(49.2)$ & $1.43(0.91-2.25)$ & 0.113 \\
\hline & TT & $78(30.6)$ & $70(27.7)$ & $148(29.1)$ & $1.11(0.68-1.83)$ & 0.661 \\
\hline \multirow[t]{3}{*}{ RARRES2/rs17173608 } & TT & $181(71.0)$ & $183(72.3)$ & $364(71.7)$ & 1.0 & \\
\hline & GT & $62(24.3)$ & $63(24.9)$ & $125(24.6)$ & $1.00(0.66-1.50)$ & 0.980 \\
\hline & GG & $12(4.7)$ & $7(2.8)$ & $19(3.7)$ & $0.57(0.22-1.49)$ & 0.253 \\
\hline \multirow[t]{3}{*}{ NOS3/rs1799983 } & GG & $144(56.5)$ & $120(47.5)$ & $264(52.0)$ & 1.0 & \\
\hline & GT & $89(34.9)$ & $119(47.0)$ & $208(40.9)$ & $1.60(1.11-2.31)$ & 0.011 \\
\hline & TT & $22(8.6)$ & $14(5.5)$ & $36(7.1)$ & $0.76(0.37-1.55)$ & 0.457 \\
\hline \multirow[t]{3}{*}{ GNB3/rs5443 } & $\mathrm{CC}$ & $27(10.6)$ & $34(13.5)$ & $61(12.0)$ & 1.0 & \\
\hline & CT & $128(50.2)$ & $124(49.0)$ & $252(49.6)$ & $0.76(0.43-1.35)$ & 0.359 \\
\hline & $\mathrm{TT}$ & $100(39.2)$ & $95(37.5)$ & $195(38.4)$ & $0.75(0.42-1.34)$ & 0.338 \\
\hline \multirow[t]{3}{*}{$A P O B / \mathrm{rs} 693$} & GG & $142(55.7)$ & $161(63.6)$ & $303(59.6)$ & 1.0 & \\
\hline & $A G$ & 89 (34.9) & $79(31.2)$ & $168(33.1)$ & $1.27(0.87-1.86)$ & 0.203 \\
\hline & AA & $24(9.4)$ & $13(5.2)$ & $37(7.3)$ & $2.09(1.02-4.26)$ & 0.038 \\
\hline \multirow[t]{3}{*}{ Alleles APOE/ rs429358 + rs7412 } & $\epsilon 2$ & $1(0.4)$ & $3(1.2)$ & $4(0.8)$ & 1.0 & \\
\hline & $\epsilon 3$ & $207(81.2)$ & $180(71.1)$ & $387(76.2)$ & $0.29(0.01-2.75)$ & 0.256 \\
\hline & $\epsilon 4$ & $47(18.4)$ & $70(27.7)$ & $117(23.0)$ & $0.49(0.02-4.82)$ & 0.541 \\
\hline \multirow[t]{6}{*}{ Genotypes APOE/ rs429358 + rs7412 } & $\mathrm{E} 2 / 2$ & $1(0.4)$ & $3(1.2)$ & $4(0.8)$ & 1.0 & \\
\hline & $E 2 / 3$ & $23(9.0)$ & $14(5.5)$ & 37 (7.3) & $0.20(0.01-2.14)$ & 0.151 \\
\hline & E2/4 & $184(72.2)$ & $166(65.6)$ & $350(68.9)$ & $0.30(0.03-2.91)$ & 0.273 \\
\hline & $E 3 / 3$ & $5(2.0)$ & $5(2.0)$ & $10(2.0)$ & $0.33(0.02-4.40)$ & 0.393 \\
\hline & $E 3 / 4$ & $40(15.6)$ & $59(23.3)$ & 99 (19.5) & $0.49(0.04-4.89)$ & 0.537 \\
\hline & E4/4 & $2(0.8)$ & $6(2.4)$ & $8(1.6)$ & $1.0(0.06-15.9)$ & 0.999 \\
\hline \multirow[t]{3}{*}{$L D L R /$ rs 5925} & $\mathrm{CC}$ & $17(6.7)$ & $15(5.9)$ & $32(6.3)$ & 1.0 & \\
\hline & $\mathrm{CT}$ & $73(28.6)$ & $68(26.9)$ & $141(27.8)$ & $0.94(0.43-2.04)$ & 0.890 \\
\hline & TT & $165(64.7)$ & $170(67.2)$ & $335(65.9)$ & $0.85(0.41-1.77)$ & 0.675 \\
\hline \multirow[t]{3}{*}{$A G T /$ rs699 } & GG & $113(44.4)$ & $113(44.7)$ & $226(44.5)$ & 1.0 & \\
\hline & $A G$ & $110(43.1)$ & $119(47.0)$ & $229(45.1)$ & $0.92(0.64-1.33)$ & 0.675 \\
\hline & AA & $32(12.5)$ & $21(8.3)$ & $53(10.4)$ & $1.52(0.82-2.80)$ & 0.173 \\
\hline \multirow[t]{3}{*}{ APOC $3 / \mathrm{rs} 5128$} & GG & $7(2.7)$ & $5(2.0)$ & $12(2.4)$ & 1.0 & \\
\hline & CG & $41(16.1)$ & $43(17.0)$ & $84(16.5)$ & $0.68(0.20-2.31)$ & 0.537 \\
\hline & $\mathrm{CC}$ & $207(81.2)$ & $205(81.0)$ & $412(81.1)$ & $0.72(0.22-2.30)$ & 0.581 \\
\hline \multirow[t]{3}{*}{ APOC3/rs4520 } & $\mathrm{CC}$ & $130(51.0)$ & $132(52.2)$ & $262(51.6)$ & 1.0 & \\
\hline & $\mathrm{CT}$ & $93(36.5)$ & $99(39.1)$ & $192(37.8)$ & $0.95(0.65-1.38)$ & 0.803 \\
\hline & TT & $32(12.5)$ & $22(8.7)$ & $54(10.6)$ & $1.47(0.81-2.67)$ & 0.196 \\
\hline
\end{tabular}

*The ancestral allele was taken for the homozygous reference group with the exception of the APOE haplotype rs429358 and rs7412 where the reference was the $\epsilon 2$ allele. ${ }^{* *} \mathrm{P}<0.05$ (Pearson's chi-squared test).

Risk genotypes were not independently associated with high levels of chemerin. However, in the concomitant presence of the AA and GT + TT genotypes of polymorphisms rs693 and rs1799983, respectively, there was a 2.21fold increase in the chance of elevated levels of chemerin (95\% Cl: $1.25-3.88)$ compared to the reference genotype (GG).

\section{Discussion}

The present study evaluated adipokine chemerin according to classical and genetic CVRFs in rural populations of Ouro Preto, Brazil. There was a significant association of classical CVRFs with high levels of chemerin. Associations with high levels of TG and IR were significant 
Table 5. Binary logistic regression analysis of risk factors of cardiovascular disease associated with high levels of chemerin in the study population.

\begin{tabular}{|c|c|c|c|c|}
\hline Variables & Crude OR $(95 \% \mathrm{Cl})$ & $\mathrm{P}$ & Adjusted $\mathrm{OR}^{*}(95 \% \mathrm{Cl})$ & $P$ \\
\hline \multicolumn{5}{|l|}{ Gender } \\
\hline Female & $2.09(1.44-3.04)$ & $<0.0001$ & $1.99(1.35-2.95)$ & 0.001 \\
\hline \multicolumn{5}{|l|}{ Age group } \\
\hline Elderly & 1.62 (1.09-2.41) & 0.015 & $1.64(1.08-2.49)$ & 0.019 \\
\hline \multicolumn{5}{|l|}{ HOMA-IR } \\
\hline Insulin resistance & 2.37 (1.40-3.95) & 0.001 & $1.82(1.03-3.22)$ & 0.038 \\
\hline \multicolumn{5}{|l|}{ TG } \\
\hline$\geqslant 150 \mathrm{mg} / \mathrm{dL}$ & $2.12(1.40-3.2)$ & $<0.0001$ & $1.91(1.23-2.98)$ & 0.004 \\
\hline \multicolumn{5}{|l|}{ rs1799983 ${ }^{a}$} \\
\hline $\mathrm{GT}+\mathrm{TT}$ & $1.43(1.01-2.03)$ & 0.041 & $1.46(1.01-2.12)$ & 0.043 \\
\hline \multicolumn{5}{|l|}{ rs $693^{b}$} \\
\hline AA & $1.39(0.97-1.98)$ & 0.068 & $1.50(1.03-2.19)$ & 0.034 \\
\hline
\end{tabular}

*Adjusted for sex, age, HOMA-IR (Homeostatic Model Assessment of Insulin Resistance), TG (triglycerides), and high-density lipoprotein cholesterol; ${ }^{a}$ reference group: genotype homozygous for the ancestral allele; ${ }^{b}$ risk group: genotype homozygous for the variant allele.

Table 6. Bivariate risk association of genotypes of the polymorphisms APOB rs693 and NOS3 rs 1799983 and levels of chemerin by quartiles in the study population.

\begin{tabular}{|c|c|c|c|c|c|c|c|}
\hline \multicolumn{2}{|c|}{ Genotypes* } & \multicolumn{2}{|c|}{ Chemerin (ng/mL) } & \multirow{2}{*}{$\begin{array}{l}\text { Crude OR } \\
(95 \% \mathrm{Cl})\end{array}$} & \multirow[t]{2}{*}{$P$} & \multirow{2}{*}{$\begin{array}{l}\text { Adjusted OR** } \\
(95 \% \mathrm{Cl})\end{array}$} & \multirow[t]{2}{*}{$P$} \\
\hline rs693 & rs1799983 & $<160(\mathrm{n}, \%)$ & $\geqslant 160(\mathrm{n}, \%)$ & & & & \\
\hline $\mathrm{GG}+\mathrm{GA}$ & GG & $56(59.6)$ & $38(40.4)$ & 1.0 & & 1.0 & \\
\hline$G G+G A$ & $\mathrm{GT}+\mathrm{TT}$ & $57(51.4)$ & $54(48.6)$ & $1.39(0.80-2.43)$ & 0.238 & $1.49(0.84-2.67)$ & 0.172 \\
\hline AA & GG & $88(51.8)$ & $82(48.2)$ & $1.37(0.82-2.28)$ & 0.222 & $1.53(0.89-2.61)$ & 0.120 \\
\hline $\mathrm{AA}$ & $\mathrm{GT}+\mathrm{TT}$ & $54(40.6)$ & $79(59.4)$ & $2.15(1.25-3.69)$ & 0.005 & $2.21(1.25-3.88)$ & 0.006 \\
\hline
\end{tabular}

*Reference genotype: rs693, GG + GA; rs1799983, GG; risk genotype: rs693, AA; rs1799983, GT+TT. *^Adjusted for sex, age, Homeostatic Model Assessment of Insulin Resistance, triglycerides, and high-density lipoprotein cholesterol.

after adjustment for confounders. The association of high levels of chemerin was also noted with the genetic polymorphisms rs693 in the $A P O B$ gene and rs1799983 in the NOS3 gene for the AA and GT genotypes, respectively. The risk-modifying model showed that the concomitant presence of genotypes AA of rs693 and GT + TT of rs 1799983 produced a 2.21 -fold increase in the chance of presenting high levels of chemerin $(95 \% \mathrm{Cl}$ : 1.25-3.88) compared to the reference genotype (GG).

The study by Bozaoglu et al. (2) has uncovered interesting aspects about chemerin, such as its association with metabolic syndrome phenotypes, obesity, $\mathrm{AH}$, dyslipidemia, and hyperglycemia. Other studies also confirmed these findings $(3,29)$. Interestingly, the average level of chemerin observed in the present study $(203.9 \mathrm{ng} / \mathrm{mL})$ was above the range considered physiological (70-150 $\mathrm{ng} / \mathrm{mL}$ ) (30). This result raised the question of whether the increased levels of chemerin in this population influenced the development of CVRFs.
In the present study, high triglyceride levels were significantly associated with high chemerin levels after risk-adjusted analysis (OR=1.91, 95\% Cl: 1.23-2.98). A strong positive correlation has been described between elevated levels of chemerin and dyslipidemia, and each component alone $(3,5,31)$. Since dyslipidemia is frequently associated with obesity and overweight, the relationship of chemerin with this metabolic disorder could be explained by mechanisms that are interconnected. A possible path would be the potential action of chemerin on lipid metabolism in the liver, skeletal muscle, and adipose tissue and the stimulation of lipolysis in adipocytes (31). Nevertheless, Becker et al. (32) did not observe alterations in the lipid profile in animal models with increased expression of chemerin. Further investigation of the influence of chemerin on lipid homeostasis is needed.

T2DM and IR were associated with high levels of chemerin in the present study. The relationship between T2DM and chemerin has not been fully elucidated, but our 
findings were in agreement with several observational studies $(3,31,33)$. Bobbert et al. (33) reported that chemerin is likely involved in the pathophysiology of T2DM. In fact, changes in chemerin levels may precede the onset of T2DM. It has been reported that chemerin regulates glucose metabolism through adipocytes in animal models and in humans $(2,3)$.

Deletion of the chemerin receptor gene (CMKLR1) showed a protective effect against obesity and decreased insulin secretion by reducing glucose uptake in skeletal muscle and adipose tissue (34). The administration of exogenous chemerin decreased tissue glucose uptake and reduced serum levels of insulin in diabetic and obese murine models, whereas glucose uptake and insulin levels did not change with exogenous chemerin in non-obese and normoglycemic models (3). Therefore, our results corroborated the mechanism proposed by other studies, reinforcing a potential association of chemerin with disorders of glucose metabolism and IR and a possible link between them and obesity. Other experimental studies should be conducted to confirm these associations.

The present study is among the pioneers showing the association between the presence of variants within the NOS3 gene ( $r$ 1799983) and the APOB gene (rs693) and elevated levels of chemerin in a mixed population in Brazil. Regarding the effects of variations in the NOS3 gene, studies indicate that decreased bioavailability of $\mathrm{NO}$ is also involved in the pathophysiology of metabolic diseases, such as T2DM and obesity. Therefore, the contribution of NOS3 gene polymorphisms, particularly the T allele of polymorphism rs1799983, to susceptibility to those conditions has been investigated (35). Interestingly, among African and Euro-American youth, carriers of the $\mathrm{T}$ allele of the same polymorphism had a higher BMI and WC (36). Another study reported that in addition to the $T$ allele $(O R=1.72 ; P=0.001)$, the presence of the TT genotype in northern Africans showed a nearly three-fold increased risk of developing obesity $(\mathrm{OR}=2.93 ; \mathrm{P}=0.03)$ (37), suggesting that this polymorphism may influence genetic susceptibility to obesity. Evidence from the Genome-wide Association Study (GWAS) points to an association of the $A P O B$ gene and its polymorphism rs693 with changes in serum lipid levels (23). However, further investigation is required to elucidate whether this variation predisposes individuals to obesity, especially in mixed populations.

\section{References}

1. WHO (World Health Organization). Global status report on noncommunicable diseases 2014. Geneva: World Health Organization; 2014.

2. Bozaoglu K, Bolton K, McMillan J, Zimmet P, Jowett J, Collier G, et al. Chemerin is a novel adipokine associated with obesity and metabolic syndrome. Endocrinology 2007; 148: 4687-4694, doi: 10.1210/en.2007-0175.
Taken together, our results provided evidence supporting the role of the variants rs 1799983 in NOS3 and rs693 in the $A P O B$ gene as modulators of chemerin through the increase in adiposity. Along these lines, increased adiposity stimulated chemerin levels to increase, which in turn induced further growth of adiposity in a positive feedback loop. More specifically, the interaction between the variants rs1799983 and rs693 may be linked to atherosclerosis and CVD by the adipokine chemerin.

The strength of this study was that it evaluated classical and emerging CVRFs in mixed rural populations, whereas few studies have investigated Brazilian populations. The sample was representative, making it possible to estimate the prevalence of the risk factors studied and ensure the external validity of the study. Nevertheless, the study had limitations. First, the cross-sectional design adopted did not allow any causal inference to be made between the factors analyzed and chemerin. Second, the sample size may have limited the results related to genetic polymorphisms. Further studies with larger samples are necessary to confirm the associations found.

In conclusion, our results indicated a high prevalence of classical CVRFs such as T2DM, overweight/obesity, dyslipidemia, and $\mathrm{AH}$ in rural populations of Ouro Preto, as well as high serum levels of the adipokine chemerin. We suggest that the development of CVRFs in this population may be influenced by two risk genotypes characteristic of variants in well-studied genes for hypertension (rs1799983) and dyslipidemia (rs693) and by chemerin as well.

\section{Acknowledgments}

The authors are grateful to the Laboratory of Clinical Analysis (LAPAC), Federal University of Ouro Preto/MG, for technical support in blood biochemistry analysis. We also thank the local health team and population for collaboration in the field work. The research was supported by the Federal University of Ouro Preto (UFOP), the National Council for Scientific and Technological Development (CNPq, process No. 306467/2018$6)$, the Foundation for Research Support of the State of Minas Gerais (FAPEMIG-APQ-02643-15), and the Coordination of Improvement of Higher Education Personnel (CAPES).

3. Ernst MC, Sinal CJ. Chemerin: at the crossroads of inflammation and obesity. Trends Endocrinol Metab 2010; 21: 660-667, doi: 10.1016/j.tem.2010.08.001.

4. İnci S, Aksan G, Doğan P. Chemerin as an independent predictor of cardiovascular event risk. Ther Adv Endocrinol Metab 2016; 7: 57-68, doi: 10.1177/204201881662 9894. 
5. Leiherer A, Muendlein A, Kinz E, Vonbank A, Rein P, Fraunberger $P$, et al. High plasma chemerin is associated with renal dysfunction and predictive for cardiovascular events - Insights from phenotype and genotype characterization. Vascul Pharmacol 2016; 77: 60-68, doi: 10.1016/ j.vph.2015.08.010.

6. Kathiresan S, Srivastava D. Genetics of human cardiovascular disease. Cell 2012; 148: 1242-1257, doi: 10.1016/ j.cell.2012.03.001.

7. de Freitas SN, Caiaffa WT, César CC, Faria VA, do Nascimento RM, Coelho GL. Nutritional risk in the urban population of Ouro Preto, southeastern region of Brazil: the Ouro Preto heart study. Arq Bras Cardiol 2007; 88: 191-199, doi: 10.1590/S0066-782X2007000200010.

8. Cândido AP, Ferreira S, Lima AA, de Carvalho Nicolato RL, de Freitas SN, Brandão $P$, et al. Lipoprotein(a) as a risk factor associated with ischemic heart disease: Ouro Preto study. Atherosclerosis 2007; 191: 454-459, doi: 10.1016/ j.atherosclerosis.2006.04.031.

9. Cândido AP, Benedetto R, Castro AP, Carmo JS, Nicolato $\mathrm{RL}$, Nascimento-Neto RM, et al. Cardiovascular risk factors in children and adolescents living in an urban area of southeast of Brazil: Ouro Preto study. Eur J Pediatr 2009; 168: 1373-13821, doi: 10.1007/s00431-009-0940-1.

10. Brasil. Ministério da Saúde. Instituto Nacional de Câncer (INCA). Coordenação de Prevenção e Vigilância (CONPREV). Abordagem e Tratamento do Fumante - Consenso 2001. Rio de Janeiro: INCA; 2001.

11. Mayfield D, McLeod G, Hall P. The CAGE questionnaire: validation of new alcoholism screning instrument. $A m \mathrm{~J}$ Psychiatry 1974; 131: 1121-1123.

12. James PA, Oparil S, Carter BL, Cushman WC, DennisonHimmelfarb C, Handler J, et al. 2014 Evidence-based guideline for the management of high blood pressure in adults: report from the panel members appointed to the Eighth Joint National Committee (JNC 8). JAMA 2014; 311: 507-520, doi: 10.1001/jama.2013.284427.

13. WHO (World Health Organization). Physical status: The use of and interpretation of anthropometry, Report of a WHO Expert Committee. Geneva: World Health Organization; 1995.

14. WHO (World Health Organization). Obesity: preventing and managing the global epidemic. Report of a WHO Consultation. WHO Technical Report Series, n. 894. Geneva: World Health Organization; 1999.

15. Vianna CA, da Silva Linhares R, Bielemann RM, Machado EC, Gonzalez-Chica DA, Matijasevich AM, et al. Accuracy and adequacy of waist circumference cut-off points currently recommended in Brazilian adults. Public Health Nutr 2014; 17: 861-869, doi: 10.1017/S1368980013000529.

16. Friedewald WT, Levy RI, Fredrickson DS. Estimation of the concentration of low-density lipoprotein cholesterol in plasma, without use of the preparative ultracentrifuge. Clin Chem 1972; 18: 499-502, doi: 10.1093/clinchem/18.6.499.

17. Expert Panel on Detection, Evaluation, and Treatment Of High Blood Cholesterol in Adults. Executive summary of the third Report of the National Cholesterol Education Program (NCEP) expert panel on detection, evaluation, and treatment of high blood cholesterol in adults (Adult Treatment Panel III). JAMA 2001; 285: 2486-2497, doi: 10.1001/jama.285. 19.2486
18. Cho NH, Shaw JE, Karuranga $S$, Huang $\mathrm{Y}$, da Rocha Fernandes JD, Ohlrogge AW, et al. IDF Diabetes Atlas: global estimates of diabetes prevalence for 2017 and projections for 2045. Diabetes Res Clin Pract 2018; 138: 271-281, doi: 10.1016/j.diabres.2018.02.023.

19. Matthews DR, Hosker JP, Rudenski AS, Naylor BA, Treacher DF, Turner RC. Homeostasis model assessment: Insulin Resistance and beta-cell function from fasting plasma glucose and insulin concentrations in man. Diabetologia 1985; 28: 412-419, doi: 10.1007/BF00280883.

20. Geloneze B, Vasques AC, Stabe CF, Pareja JC, Rosado LE, Queiroz EC, et al. HOMA1-IR and HOMA2-IR indexes in identifying insulin resistance and metabolic syndrome: Brazilian metabolic syndrome study (BRAMS). Arq Bras Endocrinol Metab 2009; 53: 281-287, doi: 10.1590/S000427302009000200020 .

21. Kimura L. Angeli CB, Auricchio MT, Fernandes GR, Pereira $A C$, Vicente JP, et al. Multilocus family-based association analysis of seven candidate polymorphisms with essential hypertension in an African-derived semi-isolated Brazilian population. Int J Hypertens, 2012; 2012: 859219, doi: 10.1155/2012/859219.

22. Mendes-Lana A, Pena GG, Freitas SN, Lima AA, Nicolato $\mathrm{RL}$, Nascimento-Neto RM, et al. Apolipoprotein E polymorphism in Brazilian dyslipidemic individuals: Ouro Preto study. Braz J Med Biol Res 2007; 40: 49-56, doi: 10.1590/ S0100-879X2007000100007.

23. Deo RC, Reich D, Tandon A, Akylbekova E, Patterson N, Waliszewska A, et al. Genetic differences between the determinants of lipid profile phenotypes in African and European Americans: the Jackson heart study. PLoS Genet 2009; 5: e1000342, doi: 10.1371/journal.pgen.1000342.

24. Dorfmeister B, Cooper JA, Stephens JW, Ireland H, Hurel SJ, Humphries SE, et al. The effect of APOA5 and APOC3 variants on lipid parameters in European Whites, Indian Asians and Afro-Caribbeans with type 2 diabetes. Biochim Biophys Acta Mol Basis Dis, 2007; 1772: 355-363, doi: 10.1016/j.bbadis.2006.11.008.

25. Song Y, Zhu L, Richa M, Li P, Yang Y, Li S. Associations of the APOC3 rs5128 polymorphism with plasma APOC3 and lipid levels: a meta-analysis. Lipids Health Dis 2015; 14: 32, doi: 10.1186/s12944-015-0027-0.

26. van Zyl T, Jerling JC, Conradie KR, Feskens EJ. Common and rare single nucleotide polymorphisms in the LDLR gene are present in a black South African population and associate with low-density lipoprotein cholesterol levels. J Hum Genet, 2014; 59: 88-94, doi: 10.1038/jhg.2013.123.

27. Passaro A, Dalla Nora E, Marcello C, Di Vece F, Morieri ML, Sanz JM, et al. PPAR $\gamma$ Pro12Ala and ACE ID polymorphisms are associated with $\mathrm{BMI}$ and fat distribution, but not metabolic syndrome. Cardiovasc Diabetol 2011; 10: 112, doi: 10.1186/1475-2840-10-112.

28. Seripa D, D'Onofrio G, Panza F, Cascavilla L, Masullo C, Pilotto A. The genetics of the human APOE polymorphism. Rejuvenation Res 2011; 14: 491-500, doi: 10.1089/rej.2011. 1169.

29. Ferland DJ, Watts SW. Chemerin: a comprehensive review elucidating the need for cardiovascular research. Pharmacol Res 2015; 99: 351-361, doi: 10.1016/j.phrs.2015.07.018.

30. Northcott JM, Yeganeh A, Taylor CG, Zahradka P, Wigle JT. Adipokines and the cardiovascular system: mechanisms 
mediating health and disease. Can J Physiol Pharmacol 2012; 90: 1029-1059, doi: 10.1139/y2012-053.

31. Zylla S, Pietzner M, Kühn JP, Völzke H, Dörr M, Nauck M, et al. Serum chemerin is associated with inflammatory and metabolic parameters-results of a population-based study. Obesity 2017; 25: 468-475, doi: 10.1002/oby.21735.

32. Becker M, Rabe K, Lebherz C, Zugwurst J, Göke B, Parhofer KG, et al. Expression of human chemerin induces Insulin Resistance in the skeletal muscle but does not affect weight, lipid levels, and atherosclerosis in LDL receptor knockout mice on high-fat diet. Diabetes 2010; 59: 28982903, doi: $10.2337 / \mathrm{db} 10-0362$.

33. Bobbert T, Schwarz F, Fischer-Rosinsky A, Maurer L, Möhlig $\mathrm{M}$, Pfeiffer AFH, et al. Chemerin and prediction of Diabetes mellitus type 2. Clin Endocrinol (Oxf) 2015; 82: 838-843, doi: 10.1111/cen.12707.

34. Ernst MC, Haidl ID, Zúñiga LA, Dranse HJ, Rourke JL, Zabel $B A$, et al. Disruption of the chemokine-like receptor-1
(CMKLR1) gene is associated with reduced adiposity and glucose intolerance. Endocrinology 2012; 153: 672-682, doi: 10.1210/en.2011-1490.

35. Oliveira-Paula GH, Lacchini R, Tanus-Santos JE. Clinical and pharmacogenetic impact of endothelial nitric oxide synthase polymorphisms on cardiovascular diseases. Nitric Oxide 2017; 63: 39-51, doi: 10.1016/j.niox.2016. 08.004.

36. Podolsky RH, Barbeau P, Kang HS, Zhu H, Treiber FA, Snieder $\mathrm{H}$. Candidate genes and growth curves for adiposity in African- and European-American youth. Int $\mathrm{J}$ Obes (Lond) 2007; 31: 1491-1499, doi: 10.1038/sj.ijo.0803673.

37. Nasr HB, Dimassi S, M'hadhbi R, Debbabi H, Kortas M, Tabka Z, et al. Functional G894T (rs1799983) polymorphism and intron-4 VNTR variant of nitric oxide synthase (NOS3) gene are susceptibility biomarkers of obesity among Tunisians. Obes Res Clin Pract 2016; 10: 465-475, doi: 10.1016/j.orcp.2015.04.008. 